\section{Propuesta para abordar los problemas de salud mental detectados en el proceso diagnóstico de la Delegación Presidencial para la reconstrucción tras el terremoto del 27 de febrero de 2010}

\author{
RODRIGO A. FIGUEROA ${ }^{1,2}$, PAULA F. CORTÉS ${ }^{2, a}$
}

\section{Proposal to address the mental health problems detected after the February 27, 2010 earthquake}

One of the most important topics mentioned by people from places affected by the February 27th, 2010 earthquake to the Presidential Delegation for the Reconstruction, was the urgent need of mental health care. Given the enormous individual and social burden of mental health sequelae after disasters, its treatment becomes a critical issue. In this article, we propose several actions to be implemented in Chile in the context of the process of recovery and reconstruction, including optimization of social communication and media response to disasters; designing and deployment of a national strategy for volunteer service; training of primary care staff in screening and initial management of post-traumatic stress reactions; and training, continuous education and clinical supervision of a critical number of therapists in evidence-based therapies for conditions specifically related to stress.

(Rev Med Chile 2016; 144: 247-252)

Key words: Disasters; Health policy; Mental health; Public health.
'Departamento de Psiquiatría, Escuela de Medicina, Pontificia Universidad Católica de Chile. Santiago, Chile.

${ }^{2}$ Centro Nacional de Investigación para la Gestión Integrada de Desastres Naturales (CIGIDEN), Comisión Nacional de Investigación Científica y Tecnológica (CONICYT), Fondo de Financiamiento de Centros de Investigación en Áreas Prioritarias (FONDAP No 15110017/2011). aPsicóloga.

Recibido el 2 de enero de 2015, aceptado el 16 de octubre de 2015.

Conflicts of interest: RA Figueroa reports personal fees from the Ministry of Health, Government of Chile, outside the submitted work. PF Cortés has nothing to declare.

Correspondencia a:

Dr. Rodrigo Figueroa

Vicuña Mackenna 4680, Macul, Santiago, Chile.

Teléfono: +56 223541801

rfiguerc@uc.cl
$\mathrm{E}$ 1 "Diagnóstico Estado de la Reconstrucción 27 de febrero de $2010^{\prime \prime}$ se enmarca en las primeras 50 medidas de reconstrucción del Gobierno de la Presidenta Michelle Bachelet ante el terremoto y tsunami del 27 de febrero de 2010 (27F). Para establecer este diagnóstico, la Delegación Presidencial para la Reconstrucción, encabezada por la Sra. Paula Forttes, hizo un recorrido por los territorios afectados, estableciendo diálogos con dirigentes, organizaciones y comunidades, así como consultando fuentes de diversos sectores con el objetivo de validar y complementar datos sectoriales y regionales en diversas materias en el plano de la reconstrucción, como vivienda, educación, salud, entre otros.

Durante las reuniones con la comunidad ex- puesta al desastre, una de las temáticas que surgió más frecuentemente fue la necesidad de contar con más y mejores servicios de apoyo en salud mental, lo que fue manifestado tanto por las autoridades de los servicios públicos como por los alcaldes, organizaciones sociales y sociedad civil de las zonas más afectadas ${ }^{1}$. Como se ha descrito ampliamente en la literatura científica ${ }^{2-5}$, luego de situaciones de desastre es habitual que algunos adultos, adolescentes y niños desarrollen secuelas psicológicas como trastorno de estrés postraumático (TEPT), depresión o trastornos de ansiedad, que podrían seguir manifestándose por años e incluso décadas después del evento ${ }^{6-8}$, lo que podría impactar de manera negativa su calidad de vida. Lo anterior es relevante desde el punto de vista comunitario, 
porque dichos trastornos, en especial el TEPT, generan una importante carga social al deteriorar el funcionamiento familiar y de pareja ${ }^{9-10}$, comunitario y laboral ${ }^{11} \mathrm{y}$ al aumentar la incidencia de suicidio $^{12}$. Así, esta clase de patología mental puede constituirse en uno de los principales obstáculos para el logro de una reconstrucción efectiva.

De las personas que desarrollan TEPT, un tercio se mantiene con síntomas después de una década ${ }^{6}$, por lo que aún existe una ventana de oportunidad para tratar a quienes desarrollaron secuelas emocionales luego del 27F y persisten con dificultades en su funcionamiento cotidiano. En esta línea, el documento "Diagnóstico Estado de la Reconstrucción Terremoto y Tsunami 27 de febrero de 2010" sugirió incorporar a la salud mental como un capítulo básico y transversal de todo el proceso de reconstrucción, no tan sólo para recuperar a las personas que quedaron con secuelas emocionales del terremoto y tsunami, sino también para fortalecer la capacidad permanente de respuesta frente al trauma, lo que ha sido considerado por algunos expertos como una oportunidad hacia la construcción de un mundo con menor miseria. La reconstrucción no sólo debería ser una oportunidad para volver a levantar lo que ya se tenía, sino que también para fijar nuevos desafíos en materia de progreso y justicia social, como ha sido propuesto por la OMS en su documento Volver a construir mejor: atención de salud mental sostenible después de una emergencia ${ }^{13}$.

Tres meses después del terremoto y tsunami del 27F, $11 \%$ de la población nacional presentaba un probable TEPT, alcanzando a más de $23 \%$ de la población en las regiones que fueron declaradas en Estado de Catástrofe. Considerando que el TEPT afectaba al 2,4\% de la población del país antes del terremoto ${ }^{14}$, este desastre produjo un aumento de la prevalencia del trastorno de $500 \%$, con mayor riesgo de desarrollo especialmente en las mujeres y en las zonas urbanas, así como en aquellas personas que el año 2009 se encontraban en condiciones de mayor pobreza, precariedad habitacional, inseguridad laboral o enfermedad (Figueroa RA, Bas C, Padilla O. Posttraumatic stress in Chile three months after the february 27th 2010 earthquake: A prepost disaster longitudinal analysis of socioeconomic risk factors in a national representative sample of 27,593 adults. Presentado en el XVI Congreso Mundial de Psiquiatría, Madrid, 14-18 de septiembre, 2014. In Abstracts
book-Regular symposia, Vol. 2: 522). Esto es consistente con la literatura internacional que muestra que la pobreza, ser mujer, estar en la edad media de la vida, haber estado más expuesto al evento, experimentar eventos secundarios posteriores al trauma y presentar otros problemas psiquiátricos previos son factores asociados a mayor riesgo ${ }^{11}$. Otro grupo importantemente afectado fueron los funcionarios de salud, que debieron enfrentar la emergencia desde dos facetas: afectados directos e intervinientes. Por ejemplo, en una muestra de funcionarios de la atención primaria de Constitución, $20 \%$ presentaba síntomas compatibles con TEPT $^{15}$. Lo anterior refleja cómo una vez más los determinantes sociales de la salud impactaron en el desarrollo de este trastorno psiquiátrico ${ }^{16}$. $\mathrm{Al}$ estar estos determinantes fuertemente vinculados a condiciones de justicia social, es un imperativo ético del Estado efectuar todas las acciones posibles para recuperar la salud mental de los afectados, especialmente de los más pobres, grupo que sistemáticamente ha enfrentado un mayor riesgo para ésta y otras patologías. Además, y dado que las secuelas psicológicas del trauma se relacionan con delincuencia y problemas sociales ${ }^{11}$, focalizarse en su tratamiento es una herramienta básica de reconstrucción y, por lo tanto, una línea de trabajo ineludible en este contexto.

Con el objetivo de aportar conceptualmente a la reflexión sobre cómo abordar los problemas detectados por la Delegación Presidencial para la Reconstrucción, en este trabajo se propone un conjunto de acciones agrupadas en diferentes niveles según el esquema previamente propuesto por Figueroa, Marín y González (2010) en el artículo Apoyo psicológico en desastres: propuesta de un modelo de atención basado en revisiones sistemáticas y metaanálisis, publicado en esta revista ${ }^{17}$. De acuerdo a este trabajo, el apoyo debería estructurarse en cinco niveles de intervención: Nivel I, difusión; Nivel II, apoyo social, Nivel III, manejo médico general, Nivel IV, manejo psiquiátrico general y Nivel V, manejo psiquiátrico por expertos. En esta propuesta no incluiremos acciones del quinto nivel porque se consideran experimentales.

\section{Nivel I}

En un primer nivel de intervención las estrategias de promoción de la salud y comunicación social planificada, dirigida, estructurada y siste- 
mática, principalmente de parte de los medios de comunicación masiva y sus actores sociales vinculados, son cruciales en las primeras semanas después de un desastre, sobre todo en su rol sobre la normalización del malestar emocional de la población y potenciales televidentes. Desde un punto de vista psiquiátrico, es esperable que la mayor parte de la población presente diversas reacciones emocionales y conductuales que no constituyen psicopatología sino más bien una reacción normal frente a una situación anormal ${ }^{18}$. En este sentido, la tarea de los medios es central, en la medida en que disminuyan la incertidumbre y los rumores a través de un aporte de información fidedigna, confiable y regular, configurándose como la principal herramienta de mitigación primaria del impacto emocional frente al desastre ${ }^{17-19}$, sumado al control del orden público y la seguridad al interior de las comunidades. Cabe destacar aquí el efecto multiplicador de la ansiedad que pueden generar la delincuencia y los saqueos, así como la repetición de imágenes del desastre a través de los medios, especialmente en los niños ${ }^{20}$. Este es un punto crítico, ya que en el documento Cobertura televisiva del terremoto: la catástrofe vista a través de la pantalla, la audiencia y la industria, del Consejo Nacional de Televisión, se señala que los televidentes se vieron impactados emocionalmente producto de la reiteración de temas e imágenes y por algunos estilos de comunicación de los hechos, principalmente testimonios audiovisuales de las víctimas, que fueron percibidos como sensacionalistas o incluso morbosos. En el mismo documento se señala como necesaria una "preparación adecuada de los futuros egresados de las escuelas de Periodismo, diseño de estrategias que permitan un buen manejo de crisis en los canales de televisión, debate público y en los medios acerca de los estándares que debieran respetarse y generación de un protocolo para la cobertura mediática en general..."21. Aquí la participación activa de las autoridades en coordinación con los medios es indispensable para que los primeros aporten un flujo continuo y regular de información que alimente con material de calidad a estos últimos. Se hace por lo tanto necesario desarrollar estrategias y mecanismos que aseguren una comunicación social adecuada y una cobertura mediática ética que no agrave la ansiedad en la población. Los esfuerzos deben estar concentrados en optimizar las estrategias y mecanismos de comunicación social en desastres, de modo de garantizar un flujo de información oficial oportuno, masivo, regular, confiable y coherente, que permita informar el devenir del desastre, aclarar precozmente rumores, educar a la población y evitar el traumatismo secundario a través de los medios. Algunas actividades que podrían ir en esta línea incluyen la sensibilización y capacitación de los diversos actores (autoridades de gobierno, editores, directores de medios, periodistas, camarógrafos, etc.), la generación de documentos guía que orienten la cobertura, la actualización del marco normativo en la materia o la entrega de reconocimientos a aquellos medios que hayan colaborado al cumplimiento de estos objetivos, entre otras iniciativas posibles.

\section{Nivel II}

En un segundo nivel, durante los primeros meses luego del desastre otro grupo fundamental de acciones de protección de la salud mental son el conjunto de labores de ayuda humanitaria que se provee directamente en los albergues, municipalidades, juntas de vecinos, ollas comunes, parroquias, sedes deportivas y otros espacios naturales de reunión social, ya sea por personal voluntario o remunerado. Este tipo de acciones contribuye a incrementar la percepción de apoyo social por parte de los afectados, lo que ha demostrado sistemáticamente estar asociado a menores niveles de TEPT ${ }^{22-24}$. En las últimas emergencias en Chile, hemos observado cómo ciertas organizaciones civiles, principalmente universitarios, motivados por la ayuda fraterna, se desplazan en masa a las zonas afectadas a colaborar en las fases de respuesta y rehabilitación brindando ayuda humanitaria entre otros servicios, lo que sin duda es un acto de generosidad valiosa que debe mantenerse en el tiempo. Sin embargo, es importante señalar que la literatura ha advertido que el trabajo del voluntariado efectuado sin una coordinación y capacitación adecuadas puede generar dificultades en los equipos de respuesta y afectar negativamente a algunas personas a través de intervenciones que pudieran ser nocivas ${ }^{25}$. Por esta razón, se hace indispensable para los próximos desastres contar con mecanismos que aseguren una ayuda del voluntariado organizada, segura y efectiva. Los llamados Primeros Auxilios Psicológicos son una herramienta valiosa para estos fines, que ha sido 
desarrollada por expertos en crisis de diversas partes del mundo y que actualmente es recomendada por la Organización Mundial de la Salud, entre otras organizaciones de renombre en gestión de crisis y emergencias ${ }^{26}$. Dada la importancia del voluntariado durante las primeras semanas de un desastre o emergencia, se propone diseñar e implementar una estrategia nacional de gestión del voluntariado en estos contextos, que permita maximizar la ayuda brindada por los voluntarios asegurando estándares de capacitación mínimos que eviten acciones nocivas no intencionadas y un trabajo coordinado que evite duplicidad de esfuerzos, vacío de funciones y accidentes. En lo particular, esta estrategia apunta a coordinar la capacitación y despliegue organizado de equipos de voluntarios (por ejemplo, estudiantes universitarios) especializados en diversas áreas de la ayuda humanitaria, lo que pudiera ser coordinado a través de diversos organismos del estado, ONG o privados (ej. INJUV, Techo, universidades e institutos, etc.).

\section{Nivel III}

Un tercer nivel de intervención de salud mental en desastres es el que se efectúa en la Atención Primaria, la que constituye un eslabón central para articular el sistema de salud comunitario. En contexto de desastres, el personal de la atención primaria, sea cual sea su formación profesional, debería ser capaz de diagnosticar y estabilizar oportuna y efectivamente a los consultantes, identificar a aquellos en riesgo de desarrollar secuelas de largo plazo, iniciar el tratamiento precoz y derivar a la atención especializada cuando corresponda. Lamentablemente el nivel de capacitación de los funcionarios de la atención primaria en manejo del trauma psicológico es escaso $^{27}$. En el escenario actual suele manejarse a las personas en situación de crisis emocional con benzodiacepinas (clonazepam, diazepam, alprazolam, etc.), las que no son recomendadas por la OMS (2013) ni por la Sociedad Internacional de Estudios Traumáticos (2008) porque podrían aumentar el riesgo de desarrollar y consolidar secuelas emocionales de largo plazo ${ }^{28-30}$. Además, es habitual que los funcionarios de la salud, dada su doble condición de afectados e intervinientes en el desastre, no se encuentren en condiciones de brindar una ayuda adecuada porque ellos mismos podrían estar sobrepasados por la emergencia ${ }^{16}$. Por lo anterior, se propone diseñar e implementar un plan permanente de capacitación en detección y manejo inicial del trauma para funcionarios de la atención primaria, el que debería asegurar que estos logren competencias para detectar precozmente a aquellas personas aquejadas de secuelas psicológicas de eventos traumáticos, brindar Primeros Auxilios Psicológicos y estabilización, evitar el uso no indicado de benzodiacepinas, iniciar el tratamiento con antidepresivos cuando corresponda y derivar adecuadamente a tratamiento psicoterapéutico. En esta línea de acción, la entrega de herramientas de autocuidado para los profesionales intervinientes es indispensable, así como el desarrollo de mecanismos que permitan el reemplazo de funcionarios cuando algunos hayan sido sobrepasados técnica y emocionalmente por la emergencia. Esta estrategia traerá beneficios no sólo para las personas afectadas por emergencias y desastres, sino también para aquellas afectadas por agresiones, asaltos, accidentes de tránsito y otras experiencias traumáticas tan comunes en la actualidad ${ }^{31}$.

\section{Nivel IV}

El cuarto nivel de intervención es aquel dirigido al tratamiento clínico especializado de las personas que quedaron con secuelas emocionales del trauma. De acuerdo a la OMS (2013) y la Sociedad Internacional de Estudios Traumáticos (2008), en una situación ideal, las intervenciones recomendadas en primera línea para el tratamiento del TEPT y otras secuelas emocionales del trauma son la Psicoterapia Cognitivo Conductual Centrada en el Trauma (TCC-CT) y la Terapia de Desensibilización y Reprocesamiento por Movimientos Oculares (EMDR, de sus siglas en inglés) ${ }^{28,29}$. Otras intervenciones comúnmente utilizadas, como la psicoterapia expresiva, la psicoterapia ecléctica, las flores de Bach, la acupuntura, el arteterapia o el psicodrama, no se encuentran en la lista de intervenciones recomendadas por dichas organizaciones $^{28,29}$. Los antidepresivos no deberían ser utilizados en primera línea para el tratamiento del TEPT, y sólo deberían administrarse cuando la TCC-CT o EMDR hayan fallado o cuando exista depresión como preexistencia o comorbilidad ${ }^{28}$. 
Actualmente la Red de Salud Mental del país no cuenta con suficientes profesionales de la salud mental entrenados en las técnicas recomendadas ni con una distribución territorial adecuada como para asegurar el acceso a cuidados especializados a toda la población, por lo que no es posible brindar la atención recomendada internacionalmente para las personas afectadas por eventos traumáticos como desastres naturales u otros. Por ejemplo, de acuerdo al registro disponible en la página web de la Asociación de EMDR Chile, sólo se encuentran acreditados 49 profesionales en el país en esta técnica, y de ellos sólo 3 atienden en regiones ${ }^{32}$. Por lo anterior se hace indispensable capacitar a una masa crítica de funcionarios de la red en intervenciones basadas en la evidencia, a través de programas de entrenamiento que habitualmente tienen una duración de $50 \mathrm{~h}$ presenciales por funcionario ${ }^{33,34}$.

En definitiva y utilizando como marco conceptual el modelo planteado por Figueroa, Marín y González $(2010)^{17}$, se proponen cuatro líneas de trabajo para reforzar el Sistema de Protección Civil y la Red de Salud Mental, lo que permitirá completar la reconstrucción emocional luego del $27 \mathrm{~F}$ y fortalecer al sistema para futuros desastres y situaciones de crisis. Se espera que todas estas líneas de acción ayuden a consolidar el proceso de reconstrucción en Chile, pero además, y quizás aún más importante, que dejen capacidades instaladas para la consolidación de una Red de Salud Mental más resolutiva.

\section{Referencias}

1. Delegación Presidencial para la Reconstrucción. Diagnóstico estado de la reconstrucción terremoto y tsunami 27 de febrero de 2010. Santiago de Chile: Gobierno de Chile; 2014 Jun. 300 p.

2. Norris FH, Friedman MJ, Watson PJ, Byrne CM, Díaz E, Kaniasty K. 60,000 disaster victims speak: Part I. An empirical review of the empirical literature, 1981-2001. Psychiatry 2002; 65 (3): 207-39.

3. Neria Y, Galea S. Mental health and disasters. Cambridge University Press; 2009.

4. North CS, Pfefferbaum B. Mental health response to community disasters: A systematic review. JAMA 2013; 310 (5): 507-18.

5. Kar N. Psychological impact of disasters on children: Review of assessment and interventions. World J Pediatr
2009; 5 (1): 5-11.

6. Green BL, Lindy JD, Grace MC, Leonard AC. Chronic posttraumatic stress disorder and diagnostic comorbidity in a disaster sample. J Nerv Ment Dis 1992; 180 (12): 760-6.

7. Yule W, Bolton D, Udwin O, Boyle S, O'Ryan D, Nurrish J. The long-term psychological effects of a disaster experienced in adolescence: I: The incidence and course of PTSD. J Child Psychol Psychiatry 2000; 41 (4): 503 11.

8. Kessler RC, Sonnega A, Bromet E, Hughes M, Nelson CB. Posttraumatic stress disorder in the national comorbidity survey. Arch Gen Psychiatry 1995; 52 (12): 1048-60.

9. Lambert JE, Engh R, Hasbun A, Holzer J. Impact of posttraumatic stress disorder on the relationship quality and psychological distress of intimate partners: A meta-analytic review. J Fam Psychol 2012; 26 (5): 729-37.

10. Chartier-Otis M, Guay S, Marchand A. Psychological and relationship distress among partners of civilian PTSD patients. J Nerv Ment Dis 2009; 197 (7): 543-6.

11. Kessler RC. Posttraumatic stress disorder: The burden to the individual and to society. J Clin Psychiatry 2000; 61 Suppl 5:4-12; discussion 13-4.

12. Wilcox HC, Storr CL, Breslau N. Posttraumatic stress disorder and suicide attempts in a community sample of urban american young adults. Arch Gen Psychiatry 2009; 66 (3): 305-11.

13. Organización Mundial de la Salud (OMS). Volver a construir mejor: atención de salud mental sostenible después de una emergencia. Información general. OMS: Ginebra, 2013.

14. Vicente B, Kohn R, Rioseco P, Saldivia S, Levav I, Torres S. Lifetime and 12-month prevalence of DSM-III-R disorders in the Chile psychiatric prevalence study. Am J Psychiatry 2006; 163 (8): 1362-70.

15. Leiva-Bianchi M. Relevancia y prevalencia del estrés post-traumático post-terremoto como problema de salud pública en Constitución, Chile. Rev Salud Pub 2011; 13 (4): 551-9.

16. Mikkonen J, Raphael D. Social Determinants of Health: The Canadian Facts. Toronto: York University School of Health Policy and Management. 2010.

17. Figueroa RA, Marín H, González M. Apoyo psicológico en desastres: Propuesta de un modelo de atención basado en revisiones sistemáticas y metaanálisis. Rev Med Chile 2010; 138 (2): 143-51.

18. Ursano RJ, Fullerton CS, Weisaeth L, Raphael B. Individual and community responses to disasters. En: Textbook of disaster psychiatry. New York: Cambridge University Press; 2007. p. 3-26. 
19. Organización Panamericana de la Salud. Gestión de la información y comunicación en emergencias y desastres: Guía para equipos de respuesta. Washington, DC. OPS, 2009.

20. Weems CF, Scott BG, Banks DM, Graham RA. Is T.V. Traumatic for all youths? The role of preexisting posttraumatic-stress symptoms in the link between disaster coverage and stress. Psychol Sci 2012; 23 (11): 1293-7.

21. Consejo Nacional de Televisión. Cobertura televisiva del terremoto: la catástrofe vista desde la pantalla, la audiencia y la industria. Santiago de Chile, julio de 2010.

22. Sugimoto T, Umeda M, Shinozaki T, Naruse T, Miyamoto Y. Sources of perceived social support associated with reduced psychological distress at one year after the great east japan earthquake: A nationwide cross-sectional survey in 2012. Psychiatry Clin Neurosci 2014, Aug 28.

23. Banks DM, Weems CF. Family and peer social support and their links to psychological distress among hurricane-exposed minority youth. Am J Orthopsychiatry 2014; 84 (4): 341-52.

24. Brewin CR, Andrews B, Valentine JD. Meta-analysis of risk factors for posttraumatic stress disorder in trauma-exposed adults. J Consult Clin Psychol 2000; 68 (5): 748-66.

25. Ministerio de Salud y Agencia de Cooperación Internacional del Japón. Voluntariado. En: Manual para la protección y cuidado de la salud mental en situaciones de emergencias y desastres. Santiago de Chile; 2011.

26. Organización Mundial de la Salud, War Trauma Foundation y Visión Mundial Internacional (2012). Primera ayuda psicológica: Guía para trabajadores de campo. OMS: Ginebra.

27. Accatino L, Figueroa RA, Montero J, González M. La preocupante falta de formación en desastres dentro de las escuelas de medicina latinoamericanas. Rev Panam Salud Publica 2010; 28 (2): 135-6.

28. World Health Organization. Guidelines for the management of conditions specifically related to stress. Geneva: WHO, 2013.

29. Foa EB, Keane TM, Friedman MJ, Cohen JA. Effective treatments for PTSD: Practice guidelines from the International Society for Traumatic Stress Studies, $2^{\text {nd }}$ Ed. New York: The Guilford Press; 2008.

30. Gelpin E, Bonne O, Peri T, Brandes D, Shalev AY. Treatment of recent trauma survivors with benzodiazepines: A prospective study. J Clin Psychiatry 1996; 57 (9): 3904.

31. Figueroa RA, Repetto P, Bernales M. Incorporación de los Primeros Auxilios Psicológicos al currículum de pregrado. Rev Med Chile 2014; 142: 934-5.

32. EMDR Chile. Terapeutas [Internet]. [cited 2015 May 29]. Available from: http://www.emdrchile.cl/terapeutas/

33. EMDRIA. Basic EMDR Training [Internet]. [cited 2015 May 29]. Available from: http://www.emdria.org/?page $=22$

34. Center for the Treatment and Study of Anxiety, Perelman School of Medicine. Intensive Training in Prolonged Exposure Therapy for PTSD [Internet]. [updated 2015; cited 2015 May 29]. Available from: http://www. med.upenn.edu/ctsa/workshops_ptsd.html 